\title{
ADAM23 is a common risk gene for canine idiopathic epilepsy
}

\author{
Lotta L. E. Koskinen 1,2,3, Eija H. Seppälä1,2,3, Jutta Weiss| ${ }^{4}$, Tarja S. Jokinen ${ }^{5}$, Ranno Viitmaa ${ }^{5}$, Reetta L. Hänninen ${ }^{1,2,3}$, \\ Pascale Quignon ${ }^{6,7}$, Andrea Fischer ${ }^{4}$, Catherine André ${ }^{6,7}$ and Hannes Lohi ${ }^{1,2,3^{*}}$
}

\begin{abstract}
Background: Idiopathic or genetic adult-onset epilepsy is a common neurological disorder in domestic dogs. Genetic association has been reported only with ADAM23 on CFA 37 in few breeds. To identify novel epilepsy genes, we performed genome-wide association (GWA) analyses in four new breeds, and investigated the association of the previously reported ADAM23 haplotype with the epilepsy phenotype in eight breeds.

Results: GWA analysis did not reveal new epilepsy loci. ADAM23 association $(p<0.05)$ was identified in five breeds. Combined analysis of all eight breeds showed significant association $\left(p=4.6 \mathrm{e}^{-6}, \mathrm{OR} 1.9\right)$.

Conclusions: Our results further support the role of ADAM23 in multiple breeds as a common risk gene for epilepsy with low penetrance. The lack of findings in the GWA analyses points towards inefficient capture of genetic variation by the current SNP arrays, causal variant(s) with low penetrance and possible phenocopies. Future work will include studies on ADAM23 function and expression in canine neurons, as well as whole-genome sequencing in order to identify additional IE genes.
\end{abstract}

Keywords: Dog, Canis familiaris, Epilepsy, Idiopathic epilepsy, ADAM23, GWA, Association

\section{Background}

Idiopathic, or primarily genetic, epilepsy (IE) is the most common neurological disease in dogs with an overall prevalence of approximately $0.62 \%$ [1]. It is common across many breeds, but some breeds have higher disease prevalence, which indicates increased genetic predisposition [1-7]. Although pedigree analysis has clearly suggested strong genetic influence, the identification of risk genes has been challenging [8].

To date, two genes underlying canine IE have been identified: LGI2 on chromosome 3 for benign juvenile epilepsy in the Lagotto Romagnolo breed [9], and ADAM23 on chromosome 37 for focal and generalized adult-onset epilepsy in the Belgian Shepherd breed [10]. According to our recent study employing high-density genome-wide association (GWA) analysis and targeted next generation sequencing in large sample cohorts,

\footnotetext{
* Correspondence: hannes.lohi@helsinki.fi

${ }^{1}$ Research Programs Unit, Molecular Neurology, University of Helsinki, Helsinki, Finland

${ }^{2}$ Department of Veterinary Biosciences and Department of Medical Genetics, University of Helsinki, Helsinki, Finland

Full list of author information is available at the end of the article
}

ADAM23 harbors a common risk haplotype for IE in Belgian Shepherd, Finnish Spitz, Schipperke and Beagle breeds [11]. The risk haplotype spans exons 5 and 11 of the ADAM23 gene and presented with odds ratios of 3.3-12.0 depending on the breed. The results suggest that $A D A M 23$ is a common but a low penetrance risk gene for IE in several breeds, but other yet unknown factors may contribute to the disease risk. ADAM23 (ADAM23 metallopeptidase domain 23) is a membraneanchored protein expressed in several tissues and involved in cell adhesion and central nervous system development. It interacts with LGI1, LGI2 and ADAM22 in synaptic transmission [12]. LGI1, LGI2 and ADAM22 have previously been implicated in epilepsy $[9,13,14]$.

In this study, we aimed to identify new IE loci by GWA analyses in four breeds: Finnish Lapphund, Kromfohrländer, Miniature Pinscher and Pyrenean Shepherd. Furthermore, we investigated the role of $A D A M 23$ in an extended epilepsy collection of altogether eight dog breeds with IE: Australian Shepherd, Irish Setter, Labrador Retriever and Whippet, and the above mentioned breeds. 


\section{Methods}

In order to identify genes underlying canine epilepsy, we investigated the characteristics of IE in the studied breeds, collected DNA samples from cases and controls with IE and performed GWA analyses in four dog breeds. We also studied the association of the ADAM23 gene with IE in eight dog breeds.

\section{Sample collection and phenotyping}

Whole blood or buccal swab samples were collected from privately-owned dogs with the owner's consent mainly from Finland but also some cases and controls from France, Germany, Sweden and UK. The samples were recruited through breed clubs, scientific collaborators, veterinarians, and advertisement in various dog fancier forums. The samples were stored at the Dog DNA bank at the University of Helsinki along with the dogs' health and pedigree information. The selection of suspected IE cases and controls was performed as described previously [11]. The suspected diagnosis of IE was based on a 10-page epilepsy questionnaire available in multiple languages (www.koirangeenit.fi) [15]. The healthy controls were not first or second degree relatives to the cases or to other controls. Genomic DNA was extracted from whole blood samples and buccal swabs using the Puregene DNA Purification Kit (Gentra Systems) and Chemagic Magnetic Separation Module I (MSM I) (Chemagen Biopolymer-Technologie AG, Baeswieler, Germany) according to the manufacturer's instructions.

\section{Clinical examination}

Altogether, 24 Kromfohrländers (17 cases, 7 controls), 12 Finnish Lapphunds (9 cases, 3 controls) and 12 Pyrenean Shepherds (10 cases, 2 controls) were clinically studied at the Referral Animal Neurology Hospital Aisti, Vantaa, Finland as described previously [7, 10]. The results of the clinical studies for Australian Shepherds have been reported previously [15]. Clinical studies were not performed for the other breeds.

\section{Genome-wide association analyses}

GWA analyses were conducted for four breeds: Finnish Lapphunds, Kromfohrländers, Miniature Pinschers and Pyrenean Shepherds. The number of samples in each breed and genotyping platforms used are presented in Table 1. The samples were genotyped either with the Affymetrix Canine Genome 2.0 Array platinum set (Affymetrix, Santa Clara, CA, USA) with $\sim 50,000$ single-nucleotide polymorphism (SNP) markers or with the Illumina Canine HD Bead chips (Illumina, Inc., San Diego, CA, USA) for more than 173,000 SNPs across all canine chromosomes. The samples were genotyped at the Centre National de Génotypage
(Paris, France) as part of the EU-funded LUPA project [16]. The genotype data was analysed with GenABEL v. 1.6-7 [17] in R v. 2.13.0. The Affymetrix GWA data was filtered for minor allele frequency $>0.05$, genotype call rate $>0.75$, individual call rate $>0.75$ and HardyWeinberg equilibrium $p>0.0001$. The Illumina GWA data was filtered for minor allele frequency $>0.05$, genotype call rate $>0.95$, individual call rate $>0.95$ and Hardy-Weinberg equilibrium $p>0.00001$. Multidimensional scaling plots were produced for each genotyped breed to identify outliers and population stratification. Identity-by-state values were calculated for each pairs of individuals to control for duplicate samples and relatedness. The GWA analyses were performed using mixed model approach in GenABEL $[17,18]$. The estimation of polygenic model was performed using the polygenic option in GenABEL [19]. If the genomic inflation factor $\lambda$ was $>1$, the association $p$-values were corrected for it. The quality control and association analyses were performed separately for each breed.

\section{ADAM23 haplotype analysis}

To replicate the previous findings in CFA37, we estimated the required sample size using the Genetic Power Calculator [20]. The parameters for power calculation derived from our previous article [10]. The statistical power of our study materials were as follows: Australian Shepherds 0.69, Finnish Lapphunds 0.55, Irish Setters 0.38, Kromfohrländers 0.43, Labrador Retrievers 0.47, Miniature Pinschers 0.22, Pyrenean Shepherds 0.26 and Whippets 0.27 . The statistical power in the whole replication material was 0.99 .

Six variants (4 SNPs and 2 indels) composing an epilepsy risk haplotype according to our previous study [11] were selected for genotyping. The genotyping was performed using competitive allele-specific PCR (KASP) assays [21] (LGC, Herts, UK) and ABI PRISM 7900HT Sequence Detection System instrument (Applied Biosystems, Foster City, CA, USA) according to the protocols provided by the manufacturers. The total call rate was 0.94, but only 0.83 in the Irish Setters. The SNPs were in HardyWeinberg equilibrium (HWE) $(p>0.05)$ in the controls except in the Labrador Retrievers the HWE $p$-values were $>0.01$. The alleles and six-variant haplotypes were analysed for association using PLINK v. 1.07 [22]. Combined association analysis was performed using the Cochran-Mantel-Haenzel-test to control for the differences between breed clusters. The associations for homozygosity were calculated using the EpiTools package in $\mathrm{R}$ (https://medepi.com/epitools/). Per individual per haplotype missingness of $<0.5$ was required in the haplotype analysis. The number of dogs included in this analysis is given in Table 2. 
Table 1 Sample numbers, GWA platforms and sample origin

\begin{tabular}{|c|c|c|c|c|c|c|c|}
\hline \multirow[b]{2}{*}{ Breed } & \multicolumn{3}{|l|}{ GWA } & \multirow[b]{2}{*}{ Platform } & \multicolumn{3}{|c|}{ ADAM23 SNP genotyping } \\
\hline & $\mathrm{N}$ cases & $\mathrm{N}$ controls & Sample origin $^{a}$ & & $\mathrm{~N}$ cases & $\mathrm{N}$ controls & Sample origin \\
\hline Australian Shepherd & na & na & na & na & 61 & 75 & GER, FIN \\
\hline Finnish Lapphund & 40 & 97 & FIN, SWE & Illumina $170 \mathrm{~K}$ & 42 & 53 & FIN, SWE \\
\hline Irish Setter (red) & na & na & na & na & 22 & 46 & FIN, UK \\
\hline Kromfohrländer & 21 & 21 & FIN & Affymetrix $50 \mathrm{~K}$ & 46 & 29 & FIN \\
\hline Labrador Retriever & na & na & na & na & 32 & 52 & FIN, GER \\
\hline Miniature Pinscher & 15 & 15 & FIN & Affymetrix $50 \mathrm{~K}$ & 16 & 15 & FIN \\
\hline Pyrenean Shepherd & 20 & 27 & FIN,FRA & Illumina $170 \mathrm{~K}$ & 18 & 23 & FIN, FRA \\
\hline Whippet & na & na & na & na & 17 & 25 & FIN, GER \\
\hline Total & 96 & 160 & & & 254 & 318 & \\
\hline
\end{tabular}

GWA genome-wide association; na: not available, ${ }^{a}$ Samples are mainly collected in these countries, FIN: Finland; FRA: France; GER: Germany; SWE: Sweden, UK: United Kingdom

\section{Results}

Epilepsy phenotypes

The main characteristics of IE of the Finnish Lapphund, Irish Setter, Kromfohrländer, Labrador Retriever, Miniature Pinscher, Pyrenean Shepherd and Whippet cohorts were collected through detailed questionnaires, and are presented in Additional file 1: Table S1. IE in the seven breeds show typical onset at early adulthood, on average at the age of 2-4.5 years (range 4 months- 8 years), and manifest with both focal and generalised seizures. A high variability in the seizure frequency is present in all breeds ranging from one reported episode in 2 years to several episodes per month. The characteristics of epilepsy of Australian Shepherds have been reported previously [15]. Besides questionnaire-based survey of the seizure history, clinical examinations were performed in selected dogs representing three breeds (Kromfohrländers, Finnish Lapphunds, Pyrenean Shepherds) to exclude possible external causes of seizures. None of the exams revealed abnormalities in the cases or controls, thereby supporting the diagnosis of IE or genetic epilepsy in those breeds.

\section{Genome wide association results}

In order to identify loci underlying canine IE, GWA analyses were performed in altogether four dog breeds: Finnish Lapphunds, Kromfohrländers, Miniature Pinschers and Pyrenean Shepherds. No associations were identified in any of the studied cohorts (Additional file 2: Figure S1).

\section{Replication of the ADAM23 association}

Single-variant association analyses showed association $(p<0.05)$ in multiple breeds in ADAM23 (Additional file 3: Table S2) with odds ratios ranging between 1.7 and 12.3 depending on the breed. The epilepsy-risk conferring alleles identified in our previous study were the major alleles in all the breeds, and they were more common among cases than controls in all breeds except for Finnish Lapphunds. A combined analysis of all breeds showed strong statistical significance $\left(p=\right.$ value $4.6 \mathrm{e}^{-6}$, OR $1.9,95 \%$ CI $1.44-2.5$ at 15,108,593 bp (genome build CanFam3.1). Combined analysis without Finnish Lapphunds showed the highest statistical significance of $p=2.5 \mathrm{e}^{-8}$ (OR 2.4, 95\% CI 1.83.3 ) at $15,108,593 \mathrm{bp}$. Association analysis combining all

Table 2 Association results of epilepsy and the ADAM23 risk haplotype ${ }^{a}$ in eight dog breeds

\begin{tabular}{|c|c|c|c|c|c|c|c|c|c|}
\hline $\begin{array}{l}\text { Breed } \\
\mathrm{N} \text { cases/controls }\end{array}$ & $\begin{array}{l}\text { Risk haplo } \\
\text { cases freq }\end{array}$ & $\begin{array}{l}\text { Risk haplo } \\
\text { control } \\
\text { freq }\end{array}$ & $p$-value & OR (95\% C.I.) & $\begin{array}{l}\text { Risk haplo } \\
\text { homozygosity } \\
\text { cases freq }\end{array}$ & $\begin{array}{l}\text { Risk haplo } \\
\text { homozygosity } \\
\text { controls freq }\end{array}$ & $p$-value & OR (95\% C.I.) & $\begin{array}{l}\text { Cases/controls } \\
\text { without risk } \\
\text { haplotype }\end{array}$ \\
\hline Australian Shepherd 59/71 & 0.66 & 0.52 & 0.03 & $1.8(1.1-3.0)$ & 0.46 & 0.32 & 0.11 & $1.8(0.9-3.6)$ & $14 \% / 28 \%$ \\
\hline Finnish Lapphund 35/50 & 0.46 & 0.50 & 0.58 & $0.8(0.5-1.6)$ & na & na & na & na & na \\
\hline Irish Setter (red) 19/36 & 0.97 & 0.90 & 0.17 & $4.0(0.5-33.7)$ & 0.95 & 0.83 & 0.22 & $3.6(0.4-32.4)$ & $0 \% / 3 \%$ \\
\hline Kromfohrländer 46/29 & 0.88 & 0.69 & 0.004 & $3.2(1.4-7.7)$ & 0.83 & 0.45 & 0.0008 & $5.5(1.9-16.8)$ & $7 \% / 7 \%$ \\
\hline Labrador Retriever 29/50 & 0.59 & 0.33 & 0.003 & $2.9(1.5-5.6)$ & 0.34 & 0.16 & 0.06 & $2.8(0.9-8.1)$ & $17 \% / 50 \%$ \\
\hline Miniature Pinscher 16/15 & 0.88 & 0.7 & 0.05 & $3.3(0.9-14.0)$ & 0.75 & 0.53 & 0.21 & $2.5(0.5-13.0)$ & $0 \% / 13 \%$ \\
\hline Pyrenean Shepherd 17/23 & 0.65 & 0.43 & 0.06 & $2.3(0.9-6.0)$ & 0.47 & 0.13 & 0.02 & $5.5(1.2-31.8)$ & $18 \% / 26 \%$ \\
\hline Whippet 17/25 & 0.94 & 0.58 & 0.0002 & $11.3(2.9-81.5)$ & 0.89 & 0.36 & 0.0005 & $12.6(2.7-102.3)$ & $0 \% / 20 \%$ \\
\hline
\end{tabular}

$P$-values $<0.05$ are bolded

OR odds ratio, C.I. confidence interval, na not analysed

${ }^{a}$ Haplotype T-C-del-del-G-G at 15,085,438 (BICF2S23030950), 15,106,446 (BICF2P1021781), 15,108,593, 15,108,802, 15,111,724 (BICF2P1131874) and 15,113,325 bp in ADAM23 ( 28 kb), respectively (CanFam 3.1$)$ 
associated breeds from our previous [11] and current studies yielded the highest statistical significance of $p=$ $4.1 \mathrm{e}^{-18}$ (OR 2.6, 95\% CI 2.1-3.2) at 15,108,593 bp.

The six-variant risk haplotype identified in our previous study [11] consisted of variants T-C-del-del-G-G at 15,085,438 (BICF2S23030950), 15,106,446 (BICF2P1021781), 15,108,593, 15,108,802, 15,111,724 (BICF2P1131874) and $15,113,325$ bp in $A D A M 23(\sim 28 \mathrm{~kb})$, respectively (CanFam3.1). This haplotype showed association $(p<0.05)$ with IE in Australian Shepherds, Kromfohrländers, Labrador Retrievers, and Whippets (Table 2). The haplotype frequencies in Pyrenean Shepherds, Miniature Pinschers and Irish Setters showed a similar trend as in the other studied breeds, although the associations were not statistically significant. The ADAM23 risk haplotype showed no evidence of association with IE in the Finnish Lapphund cohort.

\section{Discussion}

In this study, we performed GWA analyses to four dog breeds with IE in order to identify novel breed-specific high-risk loci for the disease. As we previously reported that $A D A M 23$ is an important risk gene for canine IE [10, 11], we also investigated whether $A D A M 23$ plays a role in IE in an extended epilepsy collection, which consisted of eight additional dog breeds, and altogether 573 samples.

Most common human epilepsies are thought to have complex inheritance determined by multiple susceptibility loci with or without an environmental effect [23, 24]. Our study strongly suggests that the genetic background of common adult-onset IE does not follow simple Mendelian inheritance pattern in a number of dog breeds. The lack of association findings in the GWA studies performed in four breeds, Finnish Lapphund, Kromfohrländer, Miniature Pinscher and Pyrenean Shepherd, indicates inadequate statistical power, which points towards perhaps a more complex genetic background of the disease. There is ample evidence in canine genetics, that our sample cohorts were sufficient for the detection of loci harbouring causative genes for Mendelian disorders with high penetrance and without or with only few phenocopies [9]. Also, the SNP arrays may not efficiently capture all genetic variation in at least some of the studied breeds and this may be one of the reasons for the failure to find associations [11]. For example, the best associated marker BICF2P890779 in the first study [10] does not tag the locus in all breeds. The marker associated with IE in Belgian Shepherds, Whippets and Kromfohrländers, but not in Schipperke and Finnish Spitz. However, the new six-SNP haplotype, identified by targeted resequencing, shows association in all these breeds. Other non$A D A M 23$-linked risk loci possibly exist for canine IE, but their identification will require collection of extensive sample cohorts with rigorous phenotyping as well as denser marker maps or high-throughput sequencing.
In this study, we further confirmed $A D A M 23$ as a risk gene for canine IE. Although statistical power was limited in many breed cohorts, associations were identified in several breeds: Australian Shepherd, Kromfohrländer, Labrador Retriever, Pyrenean Shepherd, and Whippet. Finnish Lapphund was the only breed, which did not show any evidence of association despite adequate statistical power. This indicates either that $A D A M 23$ does not play a role in IE risk in this particular breed, or that the linkage disequilibrium pattern at the ADAM23 locus differs in Finnish Lapphunds compared to the other studied breeds. Indeed, we may not yet have identified the causal variant in $A D A M 23$, as the almost $90 \%$ GC content of its 5 ' end has prevented us from investigating variation in $A D A M 23$ promoter region. The six variants composing the previously identified IE risk haplotype are located between exons 5 and 11 in $A D A M 23$, at least $88 \mathrm{~kb}$ away from the 5' end.

Thus far, $A D A M 23$ remains the only confirmed risk gene for the common IE in dogs, and we now have evidence for its role in IE in altogether 11 breeds. The identification of the causative variant at this locus will be included in our future experiments. In addition, we will investigate the function of ADAM23 in canine neurons in order to improve the understanding of the aetiology of canine IE. Our future experiments will also include whole-genome and whole-exome analyses of IE-affected dogs in order to identify novel genes for canine IE.

\section{Conclusions}

Idiopathic epilepsy is the most common neurological disorder in dogs. Genetic cause for the disease has been suspected in many breeds based on high disease prevalence and pedigree analyses. However, only few disease genes have been identified to date. Our results further support the role of $A D A M 23$ in multiple dog breeds as a common risk gene for epilepsy with low penetrance. The lack of findings in the GWA analyses points towards inefficient capture of genetic variation by the current SNP arrays, causal variant(s) with low penetrance and possible phenocopies. Our results warrant studies on ADAM23 function and expression in canine neurons to improve the understanding of the molecular pathogenesis of common canine epilepsy.

\section{Additional files}

Additional file 1: Table S1. Summary of the epilepsy questionnaires. (XLSX $13 \mathrm{~kb}$ )

Additional file 2: Figure S1. Genome-wide association study results. The multidimensional scaling plots (i), quantile-quantile plots (ii) and Manhattan plots (iii) are presented for Finnish Lapphunds (A), Kromfohrländers (B), Miniature Pinschers (C) and Pyrenean Shepherds (D). In the multidimensional scaling plots, red circles denote cases and blue controls. (TIF $1000 \mathrm{~kb}$ )

Additional file 3: Table S2. Allelic association results of the ADAM23 variants. (XLSX $16 \mathrm{~kb}$ ) 


\section{Acknowledgements}

Sini Karjalainen, Minna Virta and Ranja Eklund are thanked for technical assistance. Breed clubs and breeders are thanked for donating samples for the research.

\section{Funding}

This study has been partially funded by the Academy of Finland, EU FP7 LUPA, ERCStG (260997) the Jane and Aatos Erkko Foundation, the Sigrid Juselius Foundation, the University of Helsinki Research Fund, Biocentrum Helsinki and AKC Canine Health Foundation.

\section{Availability of data and materials}

The datasets analysed during the current study are available from the corresponding author on reasonable request.

\section{Authors' contributions}

LLEK participated in dog recruitment, designing and coordinating the experiments, phenotyping, sample selection and association analyses and drafted the manuscript, EHS participated in dog recruitment, designing and coordinating the experiments, phenotyping, sample selection and GWA analyses, JW participated in dog recruitment and selection, RV performed clinical studies, TSJ supervised and participated in the phenotyping, RLH participated in the genotyping experiments, $\mathrm{PQ}$ participated in dog recruitment and selection, AF supervised and participated in the dog recruitment, phenotyping and sample selection, CA participated in dog recruitment, sample selection, designing the experiments, supervision and conceiving the study, HL conceived and supervised the study, and participated in its design and coordination and helped to prepare the manuscript. All authors read and approved the final manuscript.

\section{Competing interests}

HL owns stocks from Genoscoper Oy (Ltd.) which provides genetic tests for dogs. The other authors declare that they have no competing interests.

\section{Ethics approval and consent to participate}

Collection of samples was approved by the Animal Ethics Committee at the State Provincial Office of Southern Finland (ESLH-2009-07827/Ym-23 and ESAVI/6054/04.10.03/2012), and by the CNRS ethical board, France (35-238-13). The samples were collected from privately-owned dogs with the owner's consent

\section{Author details}

${ }^{1}$ Research Programs Unit, Molecular Neurology, University of Helsinki, Helsinki, Finland. ${ }^{2}$ Department of Veterinary Biosciences and Department of Medical Genetics, University of Helsinki, Helsinki, Finland. ${ }^{3}$ Folkhälsan Institute of Genetics, Helsinki, Finland. ${ }^{4}$ Centre for Clinical Veterinary Medicine, Ludwig-Maximilians-Universität München, Munich, Germany. ${ }^{5}$ Department of Clinical Veterinary Sciences, University of Helsinki, Helsinki, Finland. ${ }^{6} \mathrm{CNRS}$, UMR 6290, Institut de Génétique et Développement de Rennes, Rennes, France. 'Université Rennes 1, UEB, Biosit, Faculté de Médecine, Rennes, France.

Received: 13 January 2016 Accepted: 27 January 2017 Published online: 31 January 2017

\section{References}

1. Kearsley-Fleet L, O'Neill DG, Volk HA, Church DB, Brodbelt DC. Prevalence and risk factors for canine epilepsy of unknown origin in the UK. Vet Rec. 2013;172(13):338

2. Famula TR, Oberbauer AM. Reducing the incidence of epileptic seizures in the Belgian Tervuren through selection. Prev Vet Med. 1998;33(1-4):251-9.

3. Berendt M, Gredal H, Pedersen LG, Alban L, Alving J. A cross-sectional study of epilepsy in Danish Labrador Retrievers: prevalence and selected risk factors. J Vet Intern Med. 2002;16(3):262-8.

4. Casal ML, Munuve RM, Janis MA, Werner P, Henthorn PS. Epilepsy in Irish Wolfhounds. J Vet Intern Med. 2006;20(1):131-5.

5. Berendt M, Gullov CH, Christensen SL, Gudmundsdottir H, Gredal H, Fredholm M, Alban L. Prevalence and characteristics of epilepsy in the Belgian shepherd variants Groenendael and Tervueren born in Denmark 1995-2004. Acta Vet Scand. 2008:50:51.

6. Gullov CH, Toft N, Baadsager MM, Berendt M. Epilepsy in the Petit Basset Griffon Vendeen: prevalence, semiology, and clinical phenotype. J Vet Intern Med. 2011;25(6):1372-8.
7. Viitmaa R, Cizinauskas S, Orro T, Niilo-Rama M, Gordin E, Lohi H, Seppälä EH, Bragge $\mathrm{H}$, Snellman M. Phenotype, inheritance characteristics, and risk factors for idiopathic epilepsy in Finnish Spitz dogs. J Am Vet Med Assoc. 2013;243(7):1001-9.

8. Ekenstedt KJ, Oberbauer AM. Inherited epilepsy in dogs. Top Companion Anim Med. 2013;28(2):51-8.

9. Seppälä EH, Jokinen TS, Fukata M, Fukata Y, Webster MT, Karlsson EK, Kilpinen SK, Steffen F, Dietschi E, Leeb T, Eklund R, Zhao X, Rilstone JJ, Lindblad-Toh K, Minassian BA, Lohi H. LGl2 truncation causes a remitting focal epilepsy in dogs. PLoS Genet. 2011;7(7):e1002194.

10. Seppälä EH, Koskinen LLE, Gullov CH, Jokinen P, Karlskov-Mortensen P, Bergamasco L, Baranowska Korberg I, Cizinauskas S, Oberbauer AM, Berendt M, Fredholm M, Lohi $\mathrm{H}$. Identification of a novel idiopathic epilepsy locus in Belgian Shepherd dogs. PLoS One. 2012;7(3):e33549.

11. Koskinen LLE, Seppälä EH, Belanger JM, Arumilli M, Hakosalo O, Jokinen P, Nevalainen EM, Viitmaa R, Jokinen TS, Oberbauer AM, Lohi H. Identification of a common risk haplotype for canine idiopathic epilepsy in the ADAM23 gene. BMC Genomics. 2015;16(1):465. -015-1651-9.

12. Sagane $K$, Ishihama $Y$, Sugimoto H. LGl1 and LGl4 bind to ADAM22, ADAM23 and ADAM11. Int J Biol Sci. 2008;4(6):387-96.

13. Kalachikov S, Evgrafov O, Ross B, Winawer M, Barker-Cummings C, Martinelli Boneschi F, Choi C, Morozov P, Das K, Teplitskaya E, Yu A, Cayanis E, Penchaszadeh G, Kottmann AH, Pedley TA, Hauser WA, Ottman R, Gilliam TC. Mutations in LGl1 cause autosomal-dominant partial epilepsy with auditory features. Nat Genet. 2002;30(3):335-41.

14. Muona M, Fukata Y, Anttonen AK, Laari A, Palotie A, Pihko $H$, Lönnqvist $T$, Valanne L, Somer M, Fukata M, Lehesjoki AE. Dysfunctional ADAM22 implicated in progressive encephalopathy with cortical atrophy and epilepsy. Neurol Genet. 2016;2(1):e46

15. Weissl J, Hülsmeyer V, Brauer C, Tipold A, Koskinen LLE, Kyöstila K, Lohi H, Sauter-Louis C, Wolf M, Fischer A. Disease progression and treatment response of idiopathic epilepsy in Australian Shepherd dogs. J Vet Intern Med. 2012;26(1):116-25.

16. Lequarre AS, Andersson L, Andre C, Fredholm M, Hitte C, Leeb T, Lohi H, Lindblad-Toh K, Georges M. LUPA: a European initiative taking advantage of the canine genome architecture for unravelling complex disorders in both human and dogs. Vet J. 2011:189(2):155-9.

17. Aulchenko YS, Ripke S, Isaacs A, van Duijn CM. GenABEL: an R library for genome-wide association analysis. Bioinformatics. 2007;23(10):1294-6.

18. Amin N, van Duijn CM, Aulchenko YS. A genomic background based method for association analysis in related individuals. PLoS One. 2007;2(12):e1274.

19. Thompson EA, Shaw RG. Pedigree analysis for quantitative traits: variance components without matrix inversion. Biometrics. 1990;46(2):399-413.

20. Purcell S, Cherny SS, Sham PC. Genetic Power Calculator: design of linkage and association genetic mapping studies of complex traits. Bioinformatics. 2003:19(1):149-50.

21. He C, Holme J, Anthony J. SNP genotyping: the KASP assay. Methods Mol Biol. 2014;1145:75-86.

22. Purcell S, Neale B, Todd-Brown K, Thomas L, Ferreira MA, Bender D, Maller J, Sklar P, de Bakker PI, Daly MJ, Sham PC. PLINK: a tool set for whole-genome association and population-based linkage analyses. Am J Hum Genet. 2007; 81(3):559-75.

23. Cavalleri GL, Weale ME, Shianna KV, Singh R, Lynch JM, Grinton B, Szoeke C, Murphy K, Kinirons P, O'Rourke D, Ge D, Depondt C, Claeys KG, Pandolfo M, Gumbs C, Walley N, McNamara J, Mulley JC, Linney KN, Sheffield LJ, Radtke RA, Tate SK, Chissoe SL, Gibson RA, Hosford D, Stanton A, Graves TD, Hanna MG, Eriksson K, Kantanen AM, Kalviainen R, O'Brien TJ, Sander JW, Duncan JS, Scheffer IE, Berkovic SF, Wood NW, Doherty CP, Delanty N, Sisodiya SM, Goldstein DB. Multicentre search for genetic susceptibility loci in sporadic epilepsy syndrome and seizure types: a case-control study. Lancet Neurol. 2007:6(11):970-80.

24. Hildebrand MS, Dahl HH, Damiano JA, Smith RJ, Scheffer IE, Berkovic SF. Recent advances in the molecular genetics of epilepsy. J Med Genet. 2013; 50(5):271-9. 